\title{
IMPLEMENTing NationWide Facility-BASEd Electronic Disease Surveillance in Sierra Leone: LESSONS LEARNED
}

Daniel W. Martin, Michelle L. Sloan, Brigette L. Gleason, Les de Wit, Mohamed Alex Vandi, David K. Kargbo, Nelson Clemens, Ansumana Kamara, Charles Njuguna, Stephen Sesay, and Tushar Singh

The Global Health Security Agenda aims to improve countries' ability to prevent, detect, and respond to infectious disease threats by building or strengthening core capacities required by the International Health Regulations (2005). One of those capacities is the development of surveillance systems to rapidly detect and respond to occurrences of diseases with epidemic potential. Since 2015, the US Centers for Disease Control and Prevention (CDC) has worked with partners in Sierra Leone to assist the Ministry of Health and Sanitation in developing an Integrated Disease Surveillance and Response (IDSR) system. Beginning in 2016, CDC, in collaboration with the World Health Organization and eHealth Africa, has supported the ministry in the development of Android device mobile data entry at the health facility for electronic IDSR (eIDSR), also known as health facility-based eIDSR. Health facility-based eIDSR was introduced via a pilot program in 1 district, and national rollout began in 2018. With more than 1,100 health facilities now reporting, the Sierra Leone eIDSR system is substantially larger than most mobile-device health (mHealth) projects found in the literature. Several technical innovations contributed to the success of health facility-based eIDSR in Sierra Leone. Among them were data compression and dual-mode (internet and text) message transmission to mitigate connectivity issues, user interface design tailored to local needs, and a continuous-feedback process to iteratively detect user or system issues and remediate challenges identified. The resultant system achieved high user acceptance and demonstrated the feasibility of an mHealth-based surveillance system implemented on a national scale.

Keywords: International Health Regulations, Communicable disease control, Population surveillance, Disease notification, Global health security implementation

Daniel W. Martin, MSPH, is an Epidemiologist, and Michelle L. Sloan, MA, is a Health Scientist; both in the Division of Global Health Protection, Center for Global Health, Centers for Disease Control and Prevention, Atlanta, GA. Brigette L. Gleason, MD, is Surveillance and Program Lead; Ansumana Kamara is a Public Health Specialist; and Tushar Singh, MD, PhD, is Country Director; all in the CDC Sierra Leone Country Office, Division of Global Health Protection, Center for Global Health, Centers for Disease Control and Prevention, Freetown, Sierra Leone. Les de Wit, MIntDev, is Information Systems Manager, Global Health Informatics, and Nelson Clemens, MBA, is eIDSR Project Coordinator, Disease Surveillance Systems; both with eHealth Africa, Freetown, Sierra Leone. Mohamed Alex Vandi, MD, is Director of Health Security and Emergencies, Ministry of Health and Sanitation, Freetown, Sierra Leone. David K. Kargbo is with the Ministry of Health and Sanitation, Freetown, Sierra Leone. Charles Njuguna, PhD, is Health Security and Emergency Cluster Lead, and Stephen Sesay is Data Manager/GIS Analyst; both with the World Health Organization, Freetown, Sierra Leone. The views expressed are the authors' own and do not necessarily represent the views of the Centers for Disease Control and Prevention, the US government, or the World Health Organization. 
$\mathrm{D}$ URING THE 2014-2016 West African Ebola epidemic, the US Centers for Disease Control and Prevention (CDC) initiated multiple activities in Sierra Leone under the Global Health Security Agenda (GHSA). ${ }^{1}$ The purpose of GHSA is to improve countries' ability to prevent, detect, and respond to infectious disease threats through core capacities required by the International Health Regulations (IHR 2005). ${ }^{2}$ GHSA efforts are categorized into 11 "action packages" that correspond to specific technical areas. ${ }^{1,3}$ In the "real-time surveillance" and "reporting" action packages, CDC and the World Health Organization (WHO) supported the Sierra Leone Ministry of Health and Sanitation (MoHS) and partners to revitalize an Integrated Disease Surveillance and Response (IDSR) system in 2015. ${ }^{4,5}$ Originally, IDSR was paper-based. The ministry and partners implemented electronic reporting, including mobile-device-based reporting, beginning in $2016 .^{6}$

The use of mobile devices for health applicationswhether for surveillance, reference, or clinical guidance-is commonly described as "mHealth." Although previous literature has documented effective implementation of mHealth for public health surveillance, ${ }^{7-11}$ including IDSR, ${ }^{12}$ most reports have described pilot programs or small-scale implementations (fewer than 50 sites). A 2013 review observed that there is "a lack of [literature documenting] mHealth applications and services operating at scale in low- and middle-income countries." 13 Based on our successful implementation of a nationwide system, this report contributes an answer to that deficit.

\section{Methods I: System Development/ IMPLEMENTATION}

Initially, IDSR was a paper-based system implemented at health facilities including hospitals and peripheral health units across Sierra Leone. An IDSR focal person at each health facility completed a paper weekly reporting form, compiling total suspected cases of 26 priority diseases or conditions (Table 1) from the health facility's clinical (paper) register. Focal persons submitted IDSR data via telephone or hard copy to the district surveillance officer at their district health management team office. District surveillance officers would compile reports from all health facilities into a weekly summary, which was submitted to the national surveillance program by email.

To increase timeliness and completeness of reporting and to facilitate analysis, the Ministry of Health and Sanitation initiated electronic IDSR (eIDSR) in 2015 with support from CDC, WHO, eHealth Africa, and other partners. eIDSR was rolled out in 2 phases:

Phase 1: Computers, internet, and data entry staff were placed in district health management teams. A national webbased surveillance database was developed on the opensource District Health Information System 2 (DHIS2), ${ }^{14}$ and district health management team personnel were trained to enter weekly data into a secure eIDSR website. Only the district team used eIDSR during Phase 1; health facility focal persons continued to use paper forms until Phase 2 .

Phase 2: eIDSR was implemented at the health facility level using Android tablets for data entry with district-level review of submissions. Health facility-based eIDSR is the first at-scale use of mobile systems for healthcare (mHealth) in Sierra Leone.

\section{eIDSR Mobile App Development}

Development of health facility-based eIDSR was overseen by a technical working group consisting of representatives from the Ministry of Health and Sanitation, CDC, WHO, and eHealth Africa. The technical working group developed initial requirements for health facility-based eIDSR (Table 2 and Figure 1). The requirements specified that the mobile application would enable data entry directly from

Table 1. Integrated Disease Surveillance and Response (IDSR) priority diseases and conditions reported weekly in Sierra Leone

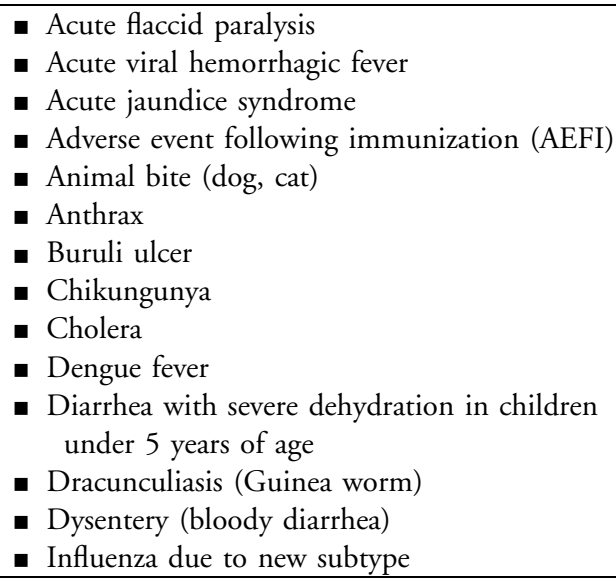

Source: Sierra Leone Technical Guidelines for Integrated Disease Surveillance and Response 
Table 2. Initial Requirements for the Health Facility-based Electronic Integrated Disease Surveillance and Response (eIDSR) Mobile Application

- Health facility-based eIDSR must transmit data into existing DHIS2-based eIDSR national system

- Use of Android devices to enable validation and message coding

- Data entry screen similar to IDSR paper forms (see Figure 1)

- Require completion of mandatory data fields

- Ability to store completed reports for later transmission
- Confirmation of entry for reports of priority diseases

- Data validation (eg, number of positive malaria tests not greater than total tests)

- Encoding of transmitted reports done by the application from plain-English data entry, rather than relying on the user to encode a report

- Capacity to transmit data by both internet (HTTP POST) and text (SMS) protocols health facilities to the national database, incorporate data validation, and have the capacity to transmit data both via internet and text messages using short message service (SMS) or short message peer-to-peer (SMPP) communication modes.

While DHIS2 remained the foundation for health facility-based eIDSR, incorporating the requirements outlined in Table 2 required substantial custom programming, including a new mechanism to receive SMS/SMPP messages. eHealth Africa performed these modifications for Sierra Leone, and all code has been submitted to DHIS2 repositories.

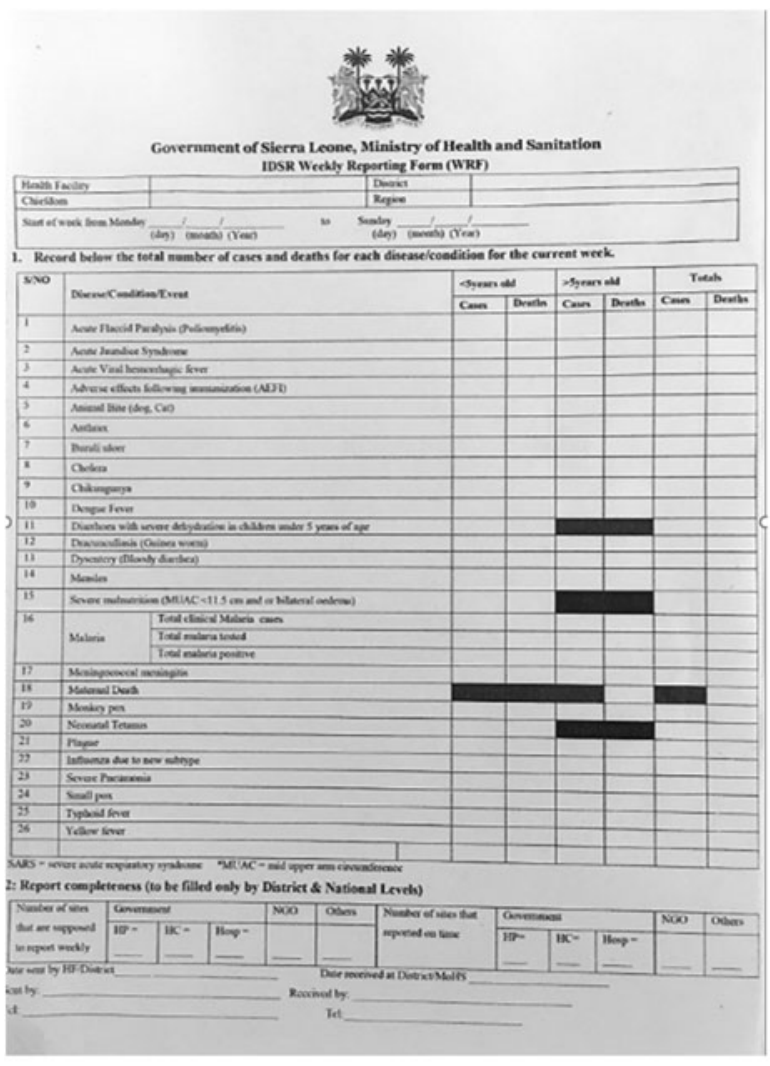

Sierra Leone Integrated Disease Surveillance \& Response (IDSR) paper reporting form, 2016
Health facility-based eIDSR was first implemented at 6 health facilities in the Port Loko district during November and December 2016. Subsequent cohorts were initiated in April and May 2017. National rollout to the remaining 13 districts began in April 2018 and was completed in June 2019.

Personnel from all IDSR-reporting health facilities were trained on eIDSR applications and procedures. They were issued tablets with software and mobile data installed. Trained health facility personnel reported in parallel, using both paper and the mobile application, and were monitored until the district achieved quality metrics set by the

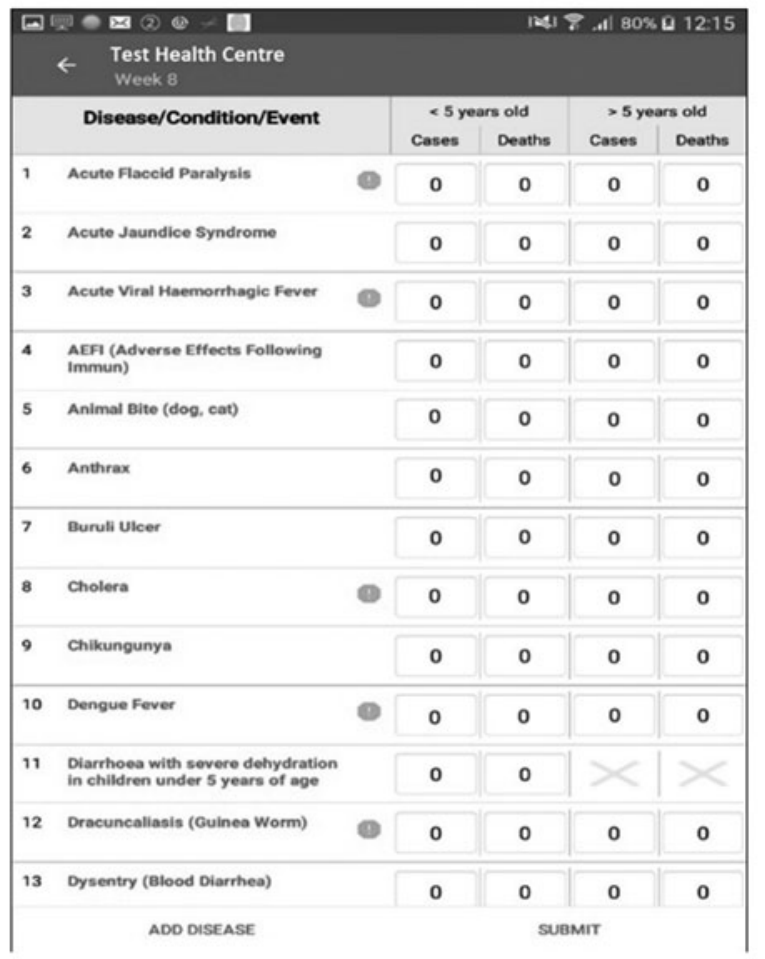

Sierra Leone electronic Integrated Disease Surveillance \& Response (eIDSR) data entry screen, 2017

Figure 1. IDSR Paper Form and Health Facility-Based eIDSR App Entry Screen, Sierra Leone, 2017 
technical working group. Once approved, health facilitybased eIDSR became the sole reporting method.

\section{Methods II: System Assessment/Data ANALYSIS}

A mixed-methods approach was employed to assess system performance. Continuous-feedback monitoring during development and throughout deployment provided rapid visibility into operational issues and permitted iterative improvements. Usability, user acceptance, and data quality were assessed among pilot users, and results were returned to the continuous-feedback process. Finally, overall system performance was assessed through centralized data analysis.

\section{Continuous Feedback}

System performance and user experience were continuously assessed through technical support calls and direct observation of users in training and at their work locations. As issues were identified, they were addressed through iterative updates to both the eIDSR application and the back-end system (Table 3).

\section{Usability/User Acceptance Assessment}

In July 2017, a usability/user-acceptance assessment was conducted in Port Loko. Of 112 health facilities that were then operational for facility-based eIDSR, 47 were assessed. Facilities were selected to represent varying levels of mobile connectivity, physical accessibility, and frequency of recent reports to the IDSR database in DHIS2.

Table 3. Continuous-Feedback Implementation for Sierra Leone Electronic Integrated Disease Surveillance \& Response (eIDSR) mobile system: Issues and Solutions

\begin{tabular}{|c|c|}
\hline Issues Identified & Solution Implemented \\
\hline $\begin{array}{l}\text { Users had difficulty understanding error messages, particularly } \\
\text { when a field was left blank or when an entry failed error } \\
\text { checks. }\end{array}$ & $\begin{array}{l}\text { Messages modified to use simplified language, to display in- } \\
\text { screen popups that require user response. Colored outlines } \\
\text { highlight the specific field in which a correction is needed. }\end{array}$ \\
\hline $\begin{array}{l}\text { Some SMS/SMPP (text) transmissions were failing mid- } \\
\text { transmission when network signals failed. This was traced to } \\
\text { the fact that reports were transmitted using } 6 \text { to } 7 \text { SMS } \\
\text { messages for a single report. }\end{array}$ & $\begin{array}{l}\text { Reporting message was compressed to a single SMS/SMPP } \\
\text { message. This also reduced data expense. }\end{array}$ \\
\hline $\begin{array}{l}\text { Users were unable to transmit SMS messages due to lack of } \\
\text { account credit. }\end{array}$ & $\begin{array}{l}\text { Implemented Short-Messaging Peer-to-Peer (SMPP) protocol, } \\
\text { which did not have a per-message charge. }\end{array}$ \\
\hline $\begin{array}{l}\text { Users did not know whether a submission was successful or not. } \\
\text { Sometimes they submitted multiple reports for the same } \\
\text { week; other times they did not re-attempt a failed transmis- } \\
\text { sion, resulting in a non-reported week. }\end{array}$ & $\begin{array}{l}\text { The eIDSR app was configured to notify the end-user of } \\
\text { successful or failed transmission. }\end{array}$ \\
\hline $\begin{array}{l}\text { Users reported weak or intermittent network signal on their } \\
\text { tablets, and they reported that phones using a different } \\
\text { mobile service provider showed a stronger signal. }\end{array}$ & $\begin{array}{l}\text { Contracts were negotiated with the } 2 \text { largest mobile providers } \\
\text { in Sierra Leone, and tablets were configured to use the } \\
\text { provider best suited to signal strength at each health facility. }\end{array}$ \\
\hline $\begin{array}{l}\text { District surveillance officers identified errors in data reported } \\
\text { from facilities in their district. }\end{array}$ & $\begin{array}{l}\text { Data review process was created to enable district surveillance } \\
\text { officers or their designees to approve all health facility } \\
\text { submissions from their district before the data are visible in } \\
\text { the national system. }\end{array}$ \\
\hline $\begin{array}{l}\text { Data quality assessment identified transcription errors between } \\
\text { paper records and tablet/DHIS } 2 \text { data. Most of these errors } \\
\text { came from entering data on the wrong line of the data entry } \\
\text { screen. }\end{array}$ & $\begin{array}{l}\text { Additional user training and clearer on-screen guidance miti- } \\
\text { gated reporting errors. }\end{array}$ \\
\hline $\begin{array}{l}\text { Data from previous reports on tablet disagreed with the DHIS2 } \\
\text { database due to correction by the district surveillance officers. } \\
\text { Users also requested ability to see their reporting history so } \\
\text { they could analyze trends in their own facilities. }\end{array}$ & $\begin{array}{l}\text { All reported data, including corrections by district surveillance } \\
\text { officers, were made available to the end-users through } \\
\text { synchronization of the mobile device with the DHIS2 } \\
\text { database. }\end{array}$ \\
\hline $\begin{array}{l}\text { Unauthorized software on tablets was corrupting the system, } \\
\text { and excessive personal use was exhausting data credit on } \\
\text { accounts. Additionally, a few tablets went missing. }\end{array}$ & $\begin{array}{l}\text { Mobile device management (MDM) was implemented to } \\
\text { restrict mobile devices to the specific applications and } \\
\text { functions needed for health facility-based eIDSR and to } \\
\text { permit remote deployment of software updates. MDM also } \\
\text { enabled device tracking and locking it down if lost or stolen. }\end{array}$ \\
\hline
\end{tabular}


Methodology consisted of a structured interview and direct observation of each user demonstrating basic operational tasks and completing a mock eIDSR report submission. The user was asked interview questions and answered using a 5-point Likert scale from "strongly agree" to "strongly disagree." For operational tasks and data entry, observers rated each user as showing no experience, novice (limited experience), intermediate (demonstrated practical ability to use the application), or expert (demonstrated full and confident ability) capacity.

Assessments were conducted at the health facility where the focal person normally worked, except for 6 health facilities that were inaccessible because of flooding during this period. On-site assessments permitted observation of functionality issues, such as app and network performance, that were not readily apparent in training locations. Issues observed were incorporated into the continuous-feedback process described above.

\section{Data Quality}

Data quality was assessed simultaneously with user acceptance. Data were collected from weekly reporting forms, health facility-based eIDSR application logs, and the national DHIS2 database. Numbers reported for suspected malaria cases and deaths from June 5 to July 2, 2017, were compared among all 3 sources. Malaria was chosen because it is the only condition for which cases reported are consistently greater than zero.

\section{System Performance}

To assess overall system performance, data on eIDSR submissions from April 9 to June 16, 2019, were exported from DHIS2 and analyzed using $\mathrm{R}$ 3.6.0. ${ }^{15}$ Communication mode for reports was categorized as manual (paper-based), mobile internet, or mobile SMS/SMPP.

\section{REsUlts}

\section{Continuous Feedback}

This process identified multiple issues with the health facilitybased eIDSR application as initially implemented. Issues detected and solutions implemented appear in Table 3.

\section{Usability and User Acceptance}

Of 47 users assessed, 43 (91\%) reported preference for tablet-based eIDSR over paper-based reporting. A total of 46 (98\%) agreed or strongly agreed with the statement, "I find the eIDSR mobile application easy to navigate," and all $47(100 \%)$ agreed or strongly agreed with the statement, "I feel very confident using the eIDSR mobile application."
Of the 47 respondents, 35 (74\%) were evaluated by the observers as expert, $6(13 \%)$ as intermediate, and $6(13 \%)$ as novice.

\section{Data Quality}

Of 188 reports assessed for data quality, 138 (74\%) had complete records in paper logs, health facility-based eIDSR application, and DHIS2. Of these 138 reports, 126 (91\%) were identical in all 3 sources, and $12(9 \%)$ had at least 1 disagreement between sources. Discordant records showed disagreement between paper-based report and health facilitybased application reporting log. Five of 138 (3.6\%) also showed disagreement between the application and DHIS2.

\section{System Performance}

As health facility-based eIDSR rolled out nationally, the proportion of IDSR submissions from health facilities through paper forms (manual) decreased and mobile submissions increased (Figure 2), while the number of facilities reporting remained roughly constant. The proportion of submissions using mobile-internet compared to mobileSMS/SMPP modes fluctuated with varying network conditions.

As of June 10, 2019, a total of 1,316 health facilities from all 14 Sierra Leone districts had been trained in health facility-based eIDSR. On that date, of these health facilities, 1,156 (88\%) submitted reports using the mobile application, 116 (9\%) by manual reporting, and 44 (3\%) failed to report. Of the 1,156 that reported through the eIDSR application, 538 (47\%) submitted by internet and $618(53 \%)$ by SMS/SMPP.

\section{Discussion}

Sierra Leone successfully implemented health facility-based electronic reporting of IDSR data on a national scale, with nearly $90 \%$ of health facilities reporting through eIDSR. In November 2016, the official Joint External Evaluation (JEE) of Sierra Leone's GHSA targets scored indicator D.2.2, an "interoperable, interconnected electronic realtime reporting system," at level 2 out of 5 (limited capacity). ${ }^{16}$ In the future, eIDSR may help Sierra Leone reach IHR compliance and potentially raise the JEE D.2.2 score.

A major strength of our assessment is that it covers the countrywide implementation of Sierra Leone's health facility-based reporting system. Multiple studies have discussed the usefulness of mHealth in improving healthcare delivery or reporting in lower-income countries, but they tend to describe small-scale interventions. ${ }^{7-12}$ With more than 1,100 health facilities in all 14 districts reporting via mobile health facility-based eIDSR, Sierra Leone has demonstrated successful scaling of an mHealth application in public health surveillance. 


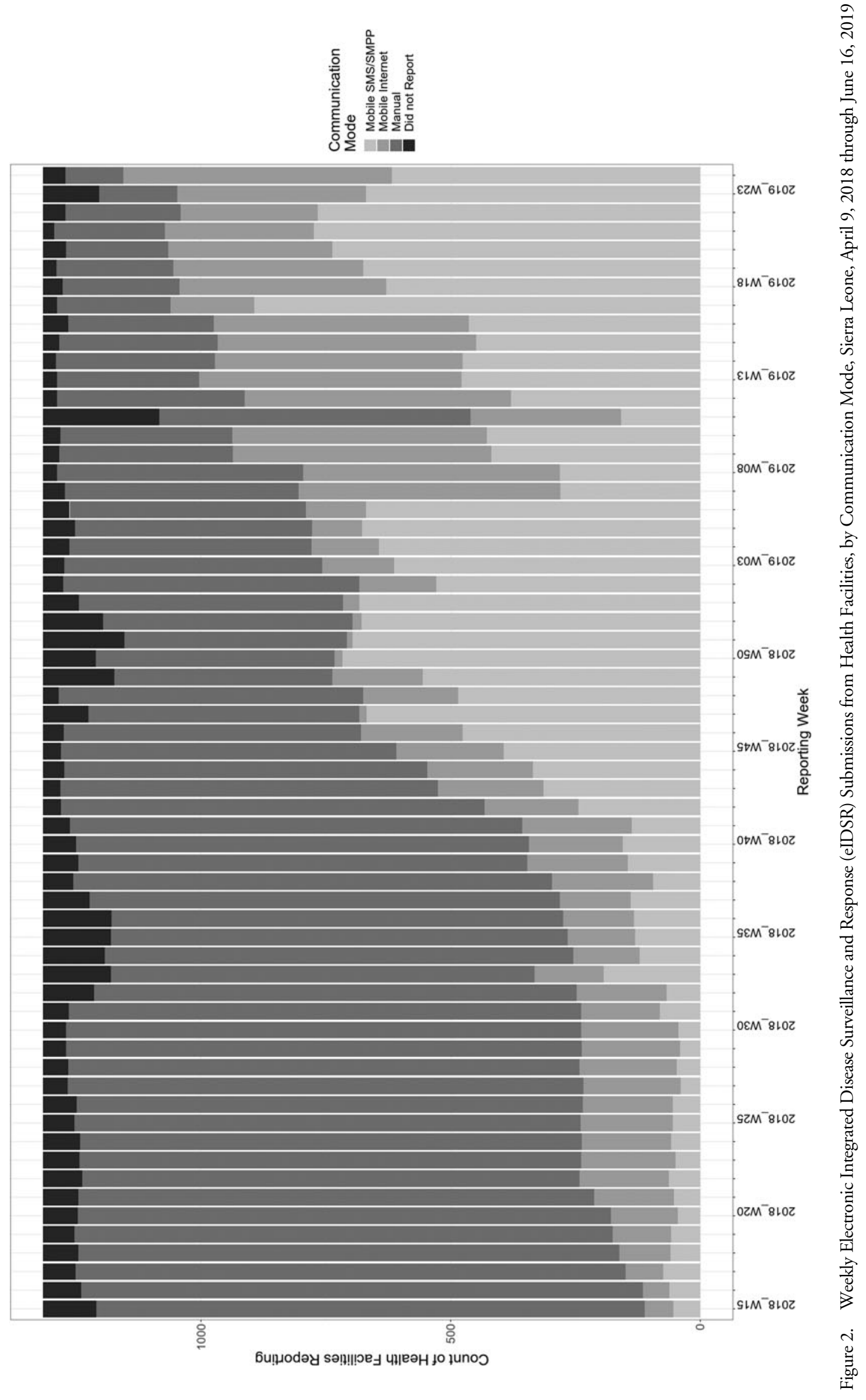

Volume 18, Number S1, 2020 
Several publications have reported health workers' ability to use mHealth applications, even with little or no prior computer experience. ${ }^{17,18}$ Our user-acceptance assessment reported high (>90\%) acceptance of the mobile eIDSR application, and over $80 \%$ of users demonstrated at least intermediate skill in its use. Braun and colleagues describe the value of in-software guidance to users. ${ }^{19}$ Through the continuous-feedback process, we observed that user-guidance and confirmation-of-submission modifications markedly improved usability of the application for health facility-based end-users.

Continuous-feedback design was vital to the success of health facility-based eIDSR and helped to identify issues of usability and function. Although not commonly described in public health literature, the principle of continuous user feedback is well known in software development. ${ }^{20-22}$ As Kazi and colleagues observed, "... iterative development with early and continuous engagement of end users ensures that the final product is efficient and usable, both key to widespread adoption." ${ }^{11}$ We concur with this observation and would add that monitoring users' experience in their actual work environments also provides vital insights into realworld operational issues and identifies needs for additional training.

The importance of improving internet connectivity for global health has been described elsewhere. ${ }^{5,23,24}$ Phalkey and colleagues ${ }^{24}$ observed that ineffective communication and transport systems adversely affect surveillance systems. Coverage throughout Sierra Leone ranges from locations with stable mobile data, to locations where only voice or SMS are accessible, to locations where there is no signal at all. In some remote health facilities, focal persons reported having to walk or ride some distance to a spot where they could obtain a signal. Furthermore, mobile connectivity in Sierra Leone is supplied by several providers, and the geographic coverage for each provider is variable. It is crucial to monitor shifts in connectivity that may happen during the life of a program, which may affect reporting rates. Mitigation strategies described here may become less important as network infrastructure improves.

Diwan and colleagues ${ }^{25}$ observed that functionality to store data for later transmission helps to address network limitations, and the Sierra Leone experience supports this observation. Our innovation in capacity to transmit by both internet and text may be a useful adaptation to consider in locations with connectivity challenges. Our results demonstrate that SMS/SMPP capacity remains an important conduit for eIDSR in Sierra Leone, even among facilities that sometimes have mobile internet access.

Our text compression modification was of vital importance to system functionality. Initially, the mobile app coded a single report into a series of 6 to 7 text messages transmitted in sequence; however, in areas with a weak signal, reports sometimes failed when not all messages were successfully transmitted. Compression to a single text message mitigated risk of transmission failure. In-app confirma- tion to users of submission success or failure and reminders for unsubmitted reports are also important.

This work is subject to a few limitations. First, this report is restricted to technical aspects of system implementation and operation and does not address eIDSR effectiveness for public health surveillance. Data quality as addressed in this report refers to completeness and concordance between data at health facilities and that reported to the system. A more robust evaluation according to CDC surveillance evaluation guidelines ${ }^{26}$ would include measures of actual disease reports and outbreaks detected. Such an evaluation would require additional on-site assessments beyond the scope of and resources available to this project and is recommended as a future strategy.

Second, usability and data quality were assessed at only 47 early-implementation health facilities, which may not be representative of all health facilities. While we believe the large scale of present implementation implies high user acceptance, additional evaluations could confirm our results and reassess data quality.

The final limitation is the question of sustainability. Continued success of health facility-based eIDSR in Sierra Leone depends on ongoing support for technical staff to maintain, update, and troubleshoot the system. Sierra Leone's technical innovations have been shared with the DHIS2 community, and incorporation into the core software may mitigate future support costs; however, continued financial support for mobile data contracts and software subscriptions is necessary.

Although the Ministry of Health and Sanitation strongly supports the eIDSR initiative, both staffing and financial requirements currently depend on external partners. ${ }^{27}$ As with any innovation in a low-resource environment, long-term ownership and sustainability depend on reconciliation of many priorities competing for limited funds.

\section{CONCLUSION}

The Sierra Leone health facility-based eIDSR system successfully receives and consolidates eIDSR reports from more than 1,100 health facilities in 14 districts. Staff at these facilities have demonstrated the ability to learn and operate tablet-based reporting software. Other countries implementing electronic surveillance or mHealth activities in resource-constrained settings may benefit from several lessons learned:

1. mHealth depends on reliable network connectivity. Adverse impacts of limited or variable connectivity can be mitigated through:

- Functionality to store data for transmission when on-network;

- Capacity to transmit data by multiple modes, such as internet and SMS/SMPP; 
- Data compression to limit size and quantity of transmitted messages; and

- Use of multiple data providers to maximize coverage.

2. An application that is easy to use is more likely to be accepted and used successfully. mHealth application design should incorporate features such as contextspecific data checks and feedback messages adapted to end-users. Error messages should highlight specific actions required and be validated through field-testing with end-users.

3. Continuous user feedback and rapid integration of modifications can enhance overall system quality and user acceptance.

The success of eIDSR in Sierra Leone demonstrates the feasibility of national-scale, facility-based, $\mathrm{mHealth}$ surveillance systems in limited-resource settings. Other countries may consider similar efforts to enhance their surveillance and reporting for global health security purposes.

\section{ACKNOWLEDGMENTS}

The authors acknowledge the contributions of Regan Hartman for manuscript review and Adaeze Ogee-Nwankwo for user-acceptance data collection. We further acknowledge the many public health professionals whose labor has supported and continues to support eIDSR in Sierra Leone.

\section{REFERENCES}

1. Global Health Security Agenda. https://www.ghsagenda.org/. Accessed February 8, 2019.

2. World Health Organization. International Health Regulations (2005). 3d ed. Geneva: WHO; 2016. https://www.who.int/ ihr/publications/9789241580496/en/. Accessed November 26, 2019.

3. Tappero JW, Cassell CH, Bunnell RE, et al. US Centers for Disease Control and Prevention and its partners' contributions to global health security. Emerg Infect Dis 2017; 23(suppl):S5-S14.

4. Government of Sierra Leone, Ministry of Health and Sanitation. Technical Guidelines for Integrated Disease Surveillance and Response in Sierra Leone. Freetown, Sierra Leone: Ministry of Health and Sanitation; 2015.

5. Njuguna C, Jambai A, Chimbaru A, et al. Revitalization of integrated disease surveillance and response in Sierra Leone post Ebola virus disease outbreak. BMC Public Health 2019; 19(1):364.

6. Marston BJ, Dokubo EK, van Steelandt A, et al. Ebola response impact on public health programs, West Africa, 2014-2017. Emerg Infect Dis 2017; 23(suppl):S25-S32.

7. Brinkel J, Krämer A, Krumkamp R, May J, Fobil J. Mobile phone-based $\mathrm{mHealth}$ approaches for public health surveil- lance in sub-Saharan Africa: a systematic review. Int J Environ Res Public Health 2014;11(11):11559-11582.

8. Rajput ZA, Mbugua S, Amadi D, et al. Evaluation of an Android-based mHealth system for population surveillance in developing countries. J Am Med Inform Assoc 2012;19(4): 655-659.

9. Githinji S, Kigen S, Memusi D, et al. Using mobile phone text messaging for malaria surveillance in rural Kenya. Malar J 2014;13:107.

10. Mtema Z, Changalucha J, Cleaveland S, et al. Mobile phones as surveillance tools: implementing and evaluating a large-scale intersectoral surveillance system for rabies in Tanzania. PLoS Med 2016;13(4):e1002002.

11. Kazi DS, Greenough PG, Madhok R, et al. Using mobile technology to optimize disease surveillance and healthcare delivery at mass gatherings: a case study from India's Kumbh Mela. J Public Health (Oxf) 2017;39(3):616-624.

12. Pascoe L, Lungo J, Kaasbøll J, Koleleni I. Collecting integrated disease surveillance and response data through mobile phones. Paper presented at: IST-Africa 2012 Conference, May 9-11, 2012; Dar es Salaam, Tanzania. http://www. ist-africa.org/home/outbox/ISTAfrica_Paper_ref_65_doc_ 4783.pdf. Accessed March 5, 2019.

13. Källander K, Tibenderana JK, Akpogheneta OJ, et al. Mobile health (mHealth) approaches and lessons for increased performance and retention of community health workers in low- and middle-income countries: a review. J Med Internet Res 2013;15(1):e17.

14. Health Information Systems Programme (HISP). Collect, manage, visualize and explore your data. DHIS2 website. https://www.dhis2.org/. Accessed February 8, 2019.

15. R Core Team. The R project for statistical computing. The R Foundation website. https://www.R-project.org/. Accessed June 17, 2019.

16. World Health Organization. Joint External Evaluation of IHR Core Capacities of the Republic of Sierra Leone. Geneva: World Health Organization; 2017. https://www.afro.who. int/publications/sierra-leone-joint-external-evaluation-ihr-corecapacity-2016. Accessed November 26, 2019.

17. Tomlinson M, Solomon W, Singh Y, et al. The use of mobile phones as a data collection tool: a report from a household survey in South Africa. BMC Med Inform Decis Mak 2009;9:51.

18. Agarwal S, Perry HB, Long LA, Labrique AB. Evidence on feasibility and effective use of mHealth strategies by frontline health workers in developing countries: systematic review. Trop Med Int Health 2015;20(8):1003-1014.

19. Braun R, Catalani C, Wimbush J, Israelski D. Community health workers and mobile technology: a systematic review of the literature. PLoS One 2013;8(6):e65772.

20. Kangas E, Kannunen T. Applying user-centered design to mobile application development. Communications of the ACM 2005;48(7):55-59.

21. de Sá M, Carrico L. Designing and evaluating mobile interaction challenges and trends. Found Trends Hum-Comput Interact 2011;4(3):175-243.

22. Dyba T, Dingsøyr T. Empirical studies of agile software development: a systematic review. Inform Softw Technol 2008; 50(9-10):833-859. 


\section{IMPLEMENTING ELECTRONIC DISEASE SURVEILLANCE IN SIERRA LEONE}

23. National Academies of Sciences, Engineering, and Medicine. Health and Medicine Division; Board on Global Health; Committee on Global Health and the Future of the United States. Global Health and the Future Role of the United States. Washington, DC: National Academies Press; 2017.

24. Phalkey RK, Yamamoto S, Awate P, Marx M. Challenges with the implementation of an Integrated Disease Surveillance and Response (IDSR) system: systematic review of the lessons learned. Health Policy Plan 2015;30(1): 131-143.

25. Diwan V, Agnihotri D, Hulth A. Collecting syndromic surveillance data by mobile phone in rural India: implementation and feasibility. Glob Health Action 2015;8:26608.

26. German RR, Lee LM, Horan JM, et al. Updated guidelines for evaluating public health surveillance systems: recom- mendations from the Guidelines Working Group. MMWR Recomm Rep 2001;50(RR-13):1-35.

27. Sloan M, Gleason B, Squire J, et al. Cost analysis of health facility electronic integrated disease surveillance and response in one district in Sierra Leone. Health Secur 2020;18(Suppl 1): 000-000.

Address correspondence to: Daniel W. Martin, MSPH Epidemiologist CGH/DGHP/GELSB Centers for Disease Control \& Prevention Atlanta, GA

Email: dwm5@cdc.gov 